products behind the flame, which have a frequency connected with the rotation of the "head " of detonation-frequencies of more than 100,000 a second being sometimes observed. The distance traversed per second by the head of detonation along its spiral path is constant in a given gas mixture, and the ratio of pitch to the diameter of tube is nearly constant, but in some cases two or more rotating ' heads ' of detonation are observable.

In the initial stages of an explosion the gaseous medium is moved forward along the direction of the tube axis : this forward impulsive motion would set up rotation about lines which may roughly be described as circles on a plane perpendicular to the axis and with centres approximately on it, and if this were superposed on an axial rotation a spiral vortex system would be initiated. Whether an explanation on this basis can be derived, or whether the rapid agitation of molecules through the gaseous medium sets up a disturbance along the axis, changing to a spiral wave front or 'head' which in turn gives rise to periodic waves in the 'tail' behind, are matters which are to be the subject of further investigations. Thirty - four photographs which Prof. Bone and Mr. Fraser have presented in their paper afford very considerable food for thought, and no such remarkable records of the behaviour of rapid flame motion have been pub. lished since those in Prof. H. B. Dixon's memoir in the Phil. Trans. of 1903.
A. C. E.

\title{
Screen-Plate Colour Photography
}

$\mathrm{T}$ HE two great lines of attack on the problem of colour photography are undoubtedly com. prised in the "subtractive" processes on one hand and the "screen-plate" processes on the other. In spite of the initial simplicity of negative-making in the subtractive process and the application to three-colour work in commercial printing, the production of the following 'positive' colour pictures by the amateur is still a matter requiring some experience, skill, and patience. Although undoubted progress has been made, the results are not of a very certain character, and they are hard to control in any precise manner.

On the other hand, the 'screen-plate' processes are of a nature much more amenable to precise control. Their disadvantage, if it be a disadvantage, is that the immediate result is a transparency rather than a picture on paper; and the transparency cannot be seen to advantage without some care in its illumination or without the use of projection apparatus. We may, however, remark that the reason why so many amateurs nowadays are dissatisfied with their photographs is because the viewing of photographs has never received a tithe of the attention which has been so lavishly given to their taking. A small print made with a 4 -inch lens is viewed from a distance of twelve or fifteen inches. The result is a false rendering of the perspective and a general impression of an insignificant little picture. If the same photograph is printed as a transparency and viewed under proper illumination with a lens of 4-inch focal length, the perspective is seen under the proper angle and the result is vastly more satisfying and impressive. The projection of the picture is an equally good alternative.

The present writer has tried in vain to convince manufacturers upon this point, but if those who produce colour transparencies take the matter to heart they are not likely to tire of their pictures, even those made on a very small scale. Properly viewed or projected, transparencies have a wealth of beauty and satisfaction to give as compared with the finest pictures on paper.

The well-known Autochrome and Agfa plates have the colour mosaic incorporated in the first layer underneath the sensitive emulsion, and the positive picture is obtained by photographic 'reversal' of the negative obtained by exposure through the mosaic. The alternative procedure uses a colour screen printed separately on another plate of glass, the screen surface being held in contact with the emulsion of a panchromatic plate during the exposure. The negative resulting on development is printed as a positive and can then be bound up with a 'viewing screen' containing a corresponding mosaic; details are generally given in articles on colour photography. In this way, any number of copies can be made.

Examples of such processes were the Joly screen plate ruled in lines, and dating from 1904, and the plates developed by Mr. C. L. Finlay, which have been embodied in the 'Thames' and 'Paget' plates. The modern form is being marketed as the 'Finlay' process.

The progress made by photographic plate manufacturers has rendered possible corresponding progress in colour photography, since it is vitally dependent on satisfactory panchromatic emulsions. An Ilford panchromatic plate $(700 \mathrm{H}$ and $\mathrm{D})$ is specially coated for the Finlay process, and a series of five carefully adjusted compensating filters is available for use with different conditions of natural and artificial lighting. The high speed of the plates allows of snapshot exposures under favourable lighting conditions if a lens working at an aperture of $f / 4 \cdot 5$, or thereabouts, is used.

The 'taking screen' with its mosaic of squares is printed by a secret process, but it is being claimed by the makers that a system of efficient inspection of the plates, and standardisation of the dyes, has enabled them to eliminate the variability which used to be experienced sometimes in the older types of plates. Methods of measurement of colour for microscopic objects are now easily available, and the efficient standardisation of the taking screens, filters, and viewing screens ought to be possible.

This is a matter of the very greatest importance for the technical use of the process in cases where pictorial colour records are required. The use of such records in microscopy, surgery, medical research, metallurgy, and other subjects would be

$$
\text { No. 3259, VoL. 129] }
$$


legion. It is impossible with a three-colour screen to make the photograph an absolutely exact rendering of the original visual impression, but it would be a great step to have a genuinely reproducible action, so that a definite colour difference occurring, say, in two photographs (taken at an interval) of a case of skin disease would have to be ascribed to an actual colour change in the object. This kind of reproducibility may be within the reach of really rigid standardisation, but this applies equally to the user's end of the business, where proper attention will be necessary in exposure, time and temperature of development and so on.

The use of a separate positive and viewing screen has now been superseded by the Finlay positive colour screen made by coating a viewing screen with a positive emulsion. Very careful registration is naturally necessary before the positive is printed, but the special system adopted in the process allows this to be done satisfactorily. The result is a picture entirely free from colour changes depending on the angle of view. It is easily understood that, granted the registration is exact, the disappearance of the parallax between the positive and the screen will result in the elimination of diffusion between the edges of the corresponding square elements in the two layers and a consequent improvement of the 'saturation" of the colour rendering. It is claimed by the makers that these plates can be used effectively for reproduction purposes for half-tone work.
Of course, the chief difficulty in making half-tone blocks from screen plates with a regular pattern has been that the pattern of the screen plate is likely to clash with that of the half-tone screen and produce moire effects. This difficulty can, however, be overcome by placing the lines of the two screens at a suitable relative angle, and using a very slight softening of the focus; it is a matter calling for some care and skill.

Development work recently in progress has been concerned with a screen-plate for photographic reversal, the application of the colour screen to kinematograph film, and the adaptation of a modified collotype process to the production of colour prints on paper, starting from the original screen-plate record. It is also claimed that it is now possible to reproduce the Finlay pictures in colour by colour gravure, collotype, and by photolitho offset. Many interesting and excellent pictures have been made for exhibition by these various processes.

In spite of difficulties and discouragements it seems that this screen-plate process should find an increasing sphere of interest and utility. Success will be the reward of a steady improvement and standardisation of the materials, and of persistent investigation of the various possibilities of the process in printing, in amateur and pro. fessional photography, and in science, but the experience of the last twenty years indicates that progress can best be made by persistent expansion from modest béginnings.

L. C. M.

\section{Industrial Bursaries *}

$I^{N}$ articles in NATURE nearly two years ago (June 21 and 28,1930 ), the late Sir Francis Ogilvie gave an interesting account of the development and work of the Royal Commission for the Exhibition of 1851 .

At that date the Commissioners had just published the Record of their Science Research Scholars, of whom the first was appointed in 1891. The Record of their Industrial Bursars, now issued, tells of a more recent branch of that work, only just alluded to by Sir Francis Ogilvie, to whose help much of its success has been due. It affords further evidence, should that be needed, of the farreaching extent and valuable results of the activities of the Commission.

Founded in 1851 to administer the surplus funds of the Exhibition, the Commissioners have for eighty years employed their resources generally in accordance with the lines laid down by the Prince Consort. "For its application", he wrote in the autumn of that year to Baron Stockmar, "I have devised a plan ", and to this plan, with such minor modifications as changing times demanded, they have adhered. It has been their object to assist in the establishment of institutions which, in the words of their Supplemental Charter, " might increase the means of Industrial Education and extend the influence of Science and Art upon productive industry".

- The Record of the Industrial Bursars of the Royal Commission for the Exhibition of 1851, 1911-1929. Published by the Commission, 1 Lowther Gardens, Exhibition Road; S.W.7.

No. 3259, VoL. 129]
In 1911 the Science Scholarships had been established for twenty years, and their value was generally recognised. They had opened a scientific career to students from all parts of the Empire, and the results achieved by those students were even then of marked importance. Now forty of their scholars are on the roll of the Royal Society.

Among the means whereby improvement and progress can be obtained in any branch of knowledge, the Prince Consort noted the "acquisition of knowledge by ocular observation, comparison, and demonstration". To make this possible for their scholars was a primary object of the Commissioners. Knowledge must first be gained and scientific investigation advanced, but this alone did not extend the influence of science on productive industry. It was with this latter object, then, that their Industrial Bursary scheme was devised - directed, as stated in their Report for 1911, to encourage the training of 'captains of industry'. At that date the engineering faculties of the universities and the technical colleges established in many large towns were well organised. Students were leaving in numbers, who might with great advantage be utilised in factories and engineering works, but they were not welcomed. It was the object of the Commission to guide in the right direction this stream, broadened by the inflow of that practical experience without which the science of the schools was of little use to the engineer, the 